\title{
Region-of-Interest Tomography for Grating-Based X-Ray Differential Phase-Contrast Imaging
}

\author{
F. Pfeiffer \\ Paul Scherrer Institut, 5232 Villigen PSI, Switzerland \\ Ecole Polytechnique Fédérale de Lausanne, 1015 Lausanne, Switzerland \\ C. David, O. Bunk, and T. Donath \\ Paul Scherrer Institut, 5232 Villigen PSI, Switzerland
}

M. Bech

Niels Bohr Institute, University of Copenhagen, 2100 Copenhagen, Denmark

G. Le Duc, A. Bravin, and P. Cloetens

European Synchrotron Radiation Facility, B.P. 220, 38043 Grenoble Cedex, France

(Received 28 April 2008; revised manuscript received 18 August 2008; published 16 October 2008)

\begin{abstract}
We report numerical and experimental results demonstrating accurate region-of-interest computed tomography (CT) reconstruction based on differential phase-contrast projection (DPC) images. The approach removes the constraint of covering the entire sample within the field of view of the image detector. Particularly for biomedical applications, the presented DPC-CT region-of-interest approach will allow for the visualization of previously inaccessible details deep inside an entire animal or organ. We envisage that this development will also be of interest for potential future clinical applications, because grating-based DPC-CT can be implemented with standard x-ray tube sources.
\end{abstract}

Absorption-based x-ray computed tomography (CT) is a well-established, nondestructive investigation method with numerous applications in life and material science. Phasecontrast computed tomography (PC-CT), which uses the phase shift rather than the absorption as the imaging signal, offers the potential of substantially increased contrast in soft tissue samples [1,2]. Several approaches for PC-CT have been developed in the past years [3-13]. One of the more recent developments is differential phase-contrast computed tomography (DPC-CT), based on a grating interferometer $[10,12,14,15]$. DPC-CT has successfully been implemented at x-ray synchrotron radiation sources for high-resolution and high-sensitivity micro-CT investigations [10,16,17]. The method is furthermore interesting for potential future medical applications of phase-contrast CT, since DPC-CT can also be implemented with more readily available $\mathrm{x}$-ray sources, such as standard $\mathrm{x}$-ray tubes [18].

That computed tomography image reconstruction based on differential phase-contrast projection data can conveniently be achieved by combining a backprojection procedure with a Hilbert filter has been demonstrated by several groups [18-20]. A major experimental constraint until now, however, is the requirement that the object has to fit entirely into the field of view covered by the image detector. This poses a severe limitation on practical applications, where, e.g., a small region of interest deep inside a large specimen is to be imaged with high resolution.

Here we report numerical and experimental results that demonstrate the feasibility of DPC-CT based on projection images that do not entirely cover the whole object. Moreover, we show that artifacts, which are known to appear in the case of conventional, absorption-based region-of-interest CT reconstructions [21], are not present in DPC-CT.

For the following, we consider a three-dimensional (3D) object described by a refractive index distribution $n(x, y, z)=1-\delta(x, y, z)+i \beta(x, y, z)$. In a conventional, absorption-based CT setup, the imaginary part $\beta$ is measured by the attenuation of the $\mathrm{x}$ rays transmitted through the specimen. A transmission projection $t_{\theta}$ in a plane defined by $z=z_{0}$ through the object can be described by combining the Radon transform of the object with BeerLambert's law [22]:

$$
t_{\theta}\left(y^{\prime}\right)=\exp \left[-\int_{-\infty}^{\infty} \frac{4 \pi}{\lambda} \beta\left(x^{\prime}, y^{\prime}\right) d x^{\prime}\right]
$$

where $x^{\prime}$ and $y^{\prime}$ denote a coordinate system which is rotated by an angle $\theta$ around the $z$ axis with respect to $x$ and $y$, respectively, and $\lambda$ the $\mathrm{x}$-ray wavelength. Note that we have omitted the variable $z$ because it does not affect the further derivation.

In differential phase-contrast imaging, one measures the effect of variations of the real part $\delta$ of the refractive index of the object by evaluating the tiny refraction angles of $\mathrm{x}$ rays induced by the specimen. Correspondingly, a differential phase-contrast projection $\alpha_{\theta}$ can be expressed by [19] 


$$
\alpha_{\theta}\left(y^{\prime}\right)=\frac{\lambda}{2 \pi} \frac{\partial \Phi_{\theta}\left(y^{\prime}\right)}{\partial y^{\prime}}=\int_{-\infty}^{\infty} \frac{\partial \delta\left(x^{\prime}, y^{\prime}\right)}{\partial y^{\prime}} d x^{\prime},
$$

where $\Phi_{\theta}\left(y^{\prime}\right)$ is the spatially dependent, total relative phase shift imprinted on the $\mathrm{x}$-ray wave front upon propagating through the specimen.

To reconstruct the original complex refractive index distribution of the object, i.e., $\beta(x, y)$ and $\delta(x, y)$, from a set of projection images described by Eqs. (1) and (2), a filtered backprojection (FBP) algorithm is usually employed [22]. In the case of the transmission projections, as obtained in conventional $\mathrm{CT}$, the reconstruction formula can be written as [22]

$$
\beta(x, y)=-\int_{0}^{\pi} \mathcal{F} \mathcal{T}^{-1}\left[\tilde{p}_{\theta}\left(\boldsymbol{v}^{\prime}\right) \tilde{k}\left(\boldsymbol{v}^{\prime}\right)\right] d \theta,
$$

where $\tilde{p}_{\theta}\left(v^{\prime}\right)$ represents the Fourier transform of the logarithm of the normalized transmission projection $t_{\theta}\left(y^{\prime}\right), \boldsymbol{v}^{\prime}$ is the Fourier space coordinate corresponding to the real space coordinate $y^{\prime}, \mathcal{F} \mathcal{T}^{-1}$ denotes the inverse Fourier transform operator, and $\tilde{k}\left(v^{\prime}\right)$ is the filter function in Fourier space. The latter is given by $\tilde{k}\left(v^{\prime}\right) \equiv\left|v^{\prime}\right|$ [23].

In the case where differential phase-contrast projections [Eq. (2)] are measured, a reconstruction of $\delta(x, y)$ is achieved by [22]

$$
\delta(x, y)=\int_{0}^{\pi} \mathcal{F} \mathcal{T}^{-1}\left[\tilde{\alpha}_{\theta}\left(\boldsymbol{v}^{\prime}\right) \tilde{h}\left(\boldsymbol{v}^{\prime}\right)\right] d \theta,
$$

where $\tilde{\alpha}_{\theta}\left(v^{\prime}\right)$ represent the Fourier transform of the projections of the measured deflection angles and $\tilde{h}=$ $1 / 2 \pi i \operatorname{sgn}\left(v^{\prime}\right)$ is the imaginary filter function for gradient projections [23].

In order to investigate the validity of the $\mathrm{CT}$ reconstructions obtained through Eqs. (3) and (4), in particular, for the case of region-of-interest reconstructions, we have carried out numerical tests. In the first step, we calculated both absorption and phase-contrast projection images for a phantom consisting of a distribution of circles [Fig. 1(a)] characterized by a refractive index of $n=1-4.26 \times$ $10^{-7}+i 1.57 \times 10^{-10}[24]$. The corresponding absorption and differential phase-contrast sinograms were calculated using an algorithm based on Eqs. (1) and (2) for an angular interval $\theta \in[0, \pi]$ and are shown in Figs. 1(b) and 1(c). The calculated sinograms consist of 1500 steps along the angular coordinate and 1024 pixels along the transverse $y^{\prime}$ coordinate. In the following step, CT reconstructions, according to Eqs. (3) and (4), were carried out and compared to the original phantom.

Figures 2(a)-2(c) display the results of the CT reconstructions of the phantom $\beta(x, y)$ distribution, obtained by applying Eq. (3) to the calculated absorption sinogram [Fig. 1(b)]. Line profiles through the center of the reconstructed (solid blue line) and the original $\beta$ values of the phantom (dashed black line) are shown in Figs. 2(d)-2(f). In Fig. 2(a) [and 2(d)], the full extent $(\gamma=1)$ of the sinogram in the $y^{\prime}$ direction was used as input for the re-
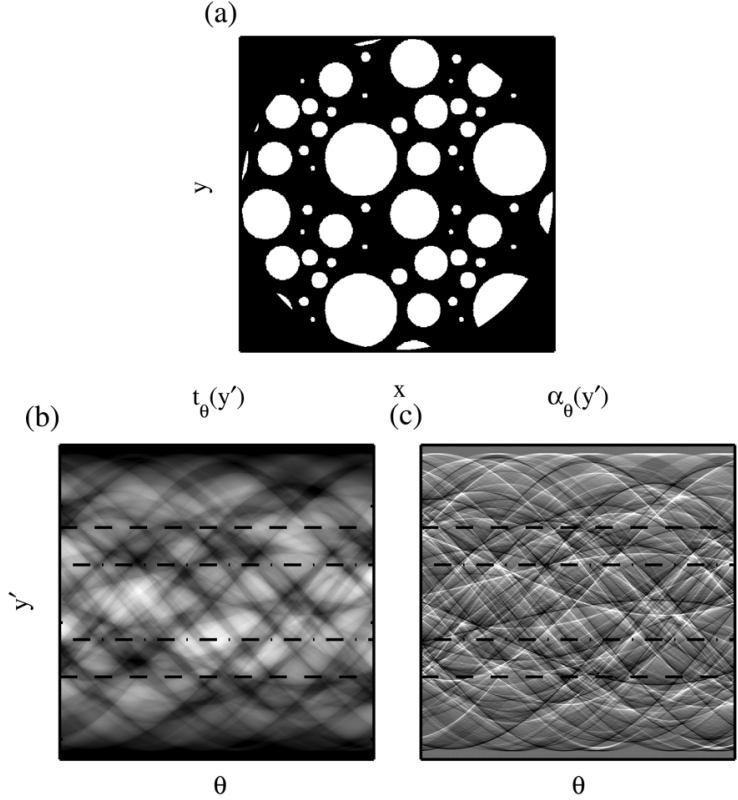

FIG. 1. (a) Schematic representation of the phantom used for the numerical simulation. The white areas denote values of $\beta=$ $1.57 \times 10^{-10}$ and $\delta=4.26 \times 10^{-7}$. (b) Calculated attenuation sinogram, as given by Eq. (1). (c) Calculated phase gradient sinogram, as given by Eq. (2). The dashed-dotted and dashed lines mark the selected projection range for the region-of-interest reconstructions displayed in Figs. 2(b), 2(c), 3(b), and 3(c).

construction, whereas Fig. 2(b) [and 2(e)] and Fig. 2(c) [and 2(f)] represent results where only the center half $(\gamma=$ $0.5)$ or quarter $(\gamma=0.25)$ was used. We observe that, apart from some high frequency noise induced by the FBP (a)

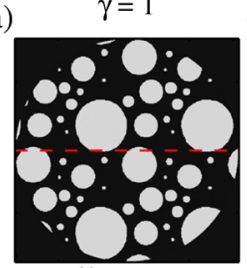

(d) $\times 10^{-1}$

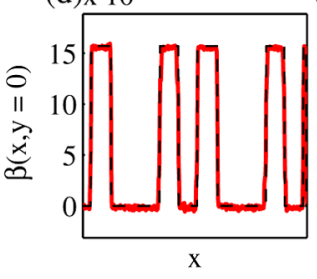

(b)

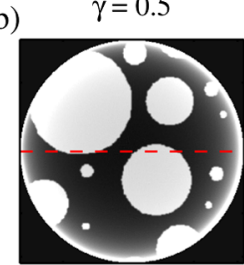

(e)

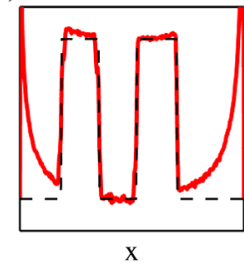

(c) $\gamma=0.25$

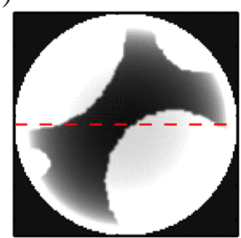

(f)

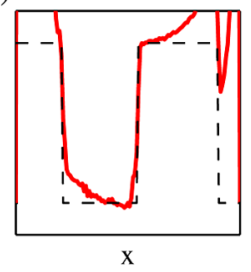

FIG. 2 (color online). (a)-(c) CT reconstructions of the phantom $\beta(x, y)$ distribution from the calculated absorption sinogram [Fig. 1(b)], using Eq. (3). (d)-(f) Line profiles through the center of the reconstructed (solid red line) and original $\beta$ values (dashed black line). In (a),(d), the full extent $(\gamma=1)$ of the sinogram in the $y^{\prime}$ direction was used as input for the CT reconstruction, whereas (b),(e) and (c),(f) represent results where only the center half $(\gamma=0.5)$ or quarter $(\gamma=0.25)$ was used. 
algorithm, the phantom is correctly reconstructed when the full sinogram is used, i.e., when the sample is entirely contained in the field of view [ $\gamma=1$, Figs. 2(a) and 2(d)]. In the cases where truncated projections $(\gamma<1)$ are used as input for the FBP, low spatial frequency artifacts occur in the corresponding reconstructions [see Fig. 2(b) or 2(e) and 2(c) or 2(f)]. These artifacts, which increasingly harm the reconstruction with increasing distance from the center of rotation, are well-known and reported in the literature (see, for example, [25]). These artifacts basically stem from the fundamental mathematical properties of the Radon transform that cause a nonlocality in the case of absorptionbased $\mathrm{CT}$ reconstructions [21,25].

In contrast to this, we do not observe these artifacts in the case of CT reconstructions based on differential phasecontrast projections. This is shown in Fig. 3, where panels (a)-(c) display DPC-CT reconstructions of the phantom $\delta(x, y)$ distribution, obtained by applying Eq. (4) to the calculated phase-contrast sinogram [Fig. 1(c)]. Additional line profiles through the center of the reconstructed (solid blue line) and original $\delta$ values (dashed black line) are shown in Figs. 3(d)-3(f). In Fig. 3(a) [and 3(d)], the full extent $(\gamma=1)$ of the sinogram in the $y^{\prime}$ direction was used as input for the CT reconstruction, whereas Fig. 3(b) [and 3(e)] and Fig. 3(c) [and 3(f)] represent results where only the center half $(\gamma=0.5)$ or quarter $(\gamma=0.25)$ was used. We observe that, apart from some high spatial frequency noise induced by the FBP algorithm, the phantom is correctly reconstructed in all cases, even for $\gamma<1$, i.e., also in the cases where the sample is not entirely contained in the field of view. These findings are in agreement with what was predicted theoretically $[26,27]$ and demonstrated (a)

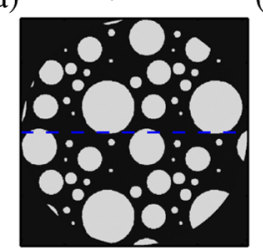

(d) $\times 10^{-7}$

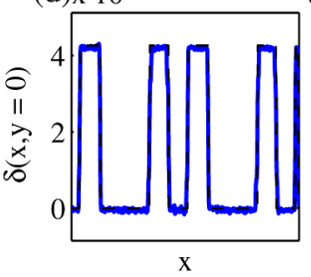

(b)

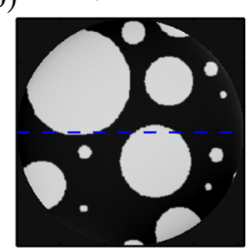

(e)

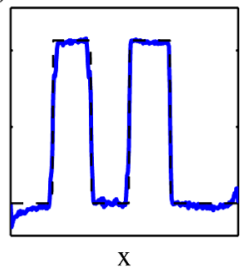

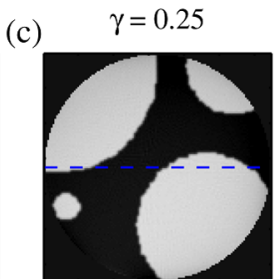

(f)

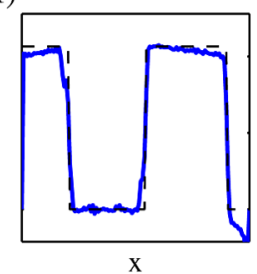

FIG. 3 (color online). (a)-(c) CT reconstructions of the phantom $\delta(x, y)$ distribution from the calculated phase-contrast sinogram [Fig. 1(c)], using Eq. (4). (d)-(f) Line profiles through the center of the reconstructed (solid blue line) and original $\delta$ values (dashed black line). In (a),(d), the full extent $(\gamma=1)$ of the sinogram in the $y^{\prime}$ direction was used as input for the CT reconstruction, whereas (b),(e) and (c),(f) represent results where only the center half $(\gamma=0.5)$ or quarter $(\gamma=0.25)$ was used. for the case of propagation-based (inline) phase-contrast imaging in Ref. [21].

We experimentally verified our conclusions drawn from the numerical studies presented further above by reconstructing regions of interests from measured DPC sinograms [see Fig. 4(a)]. The experimental data set used for the reconstruction was recorded in an imaging experiment carried out at the beam line ID19 of the European Synchrotron Radiation Facility (ESRF, Grenoble) using a two-grating interferometer [17]. The specimen was a rat brain fixed in a $4 \%$ formalin solution. A monochromatic x-ray beam of $24.9 \mathrm{keV}(\lambda=0.498 \AA)$ was used for the measurements. The images were recorded using a $15 \mu \mathrm{m}$ thick polycrystalline gadolinium oxysulphide scintillation screen with a magnifying optical lens system and a cooled charge coupled device (CCD) [28]. The full field of view was $16.1 \times 16.1 \mathrm{~mm}^{2}$. For the acquisition of a full tomographic data set, the object was rotated around the tomographic rotation axis and differential phase-contrast projection images were recorded for each projection angle [29]. In total, 721 differential phase-contrast projections over an angular range of $\theta \in[0,2 \pi]$ were recorded (see $[17,30]$ for further details on the experimental setup). (a)

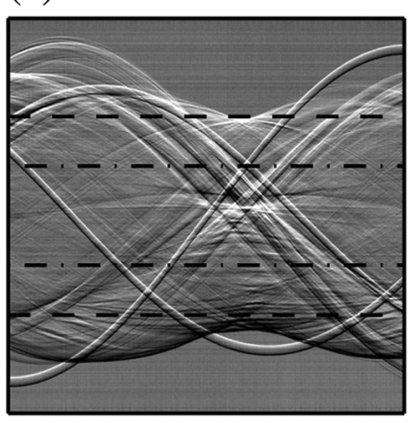

(c) $\delta(x, y), \gamma=0.5$

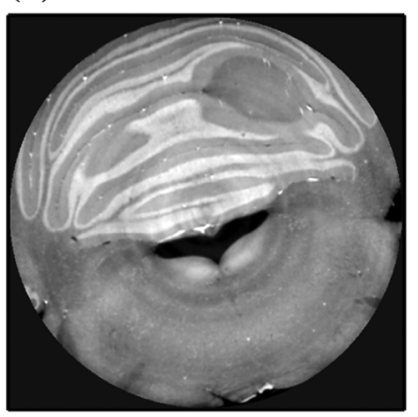

(b) $\delta(x, y), \gamma=1$

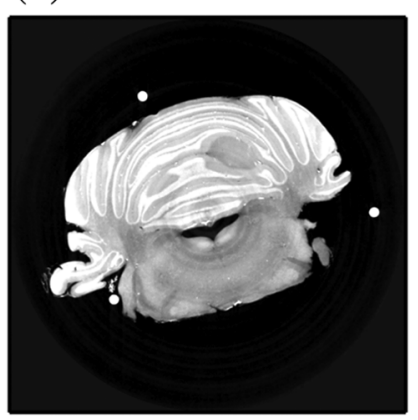

(d) $\delta(x, y), \gamma=0.25$

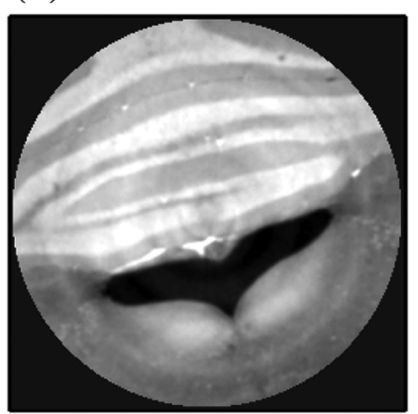

FIG. 4. (a) Measured differential phase-contrast projection sinogram of a rat brain specimen $(\theta \in[0, \pi])$. (b) Full DPC-CT reconstruction and (c),(d) region-of-interest DPC-CT reconstruction of the specimen $\delta(x, y)$ distribution using Eq. (4). In (b), the full extent $(\gamma=1)$ of the sinogram in the $y^{\prime}$ direction was used as input for the DPC-CT reconstruction, whereas (c),(d) represent results where only the center half $(\gamma=0.5)$ or the center quarter $(\gamma=0.25)$ was used. 
Figure 4(b) displays a DPC-CT reconstruction of $\delta$ using the full data set $(\gamma=1)$. In this case, the specimen is fully contained in the field of view of the image detector, for all angular projections. Figures 4(c) and 4(d) show reconstructions where only the center half [the region between the two dashed lines in Fig. 4(a)] or the center quarter [the region between the two dashed-dotted lines in Fig. 4(a)] was used as input for Eq. (4). In both case, where $\gamma<1$, we do not observe any artifacts from the truncation of the sinograms.

In summary we have demonstrated, both numerically and with real experimental data, that artifact-free regionof-interest tomography reconstructions based on differential phase-contrast projection images are possible. This procedure removes the constraint of fully covering the whole sample within the field of view given by the detector. We envisage that, particularly for medical applications, where phase-contrast DPC-CT has been proven to be a uniquely powerful method, a region-of-interest approach will allow for the visualization of previously inaccessible, high-resolution details deep inside a whole animal or organ. In such a way, micron-resolved three-dimensional imaging of, e.g., the detailed blood-vessel network structure in a living rat or mouse can be envisioned in the future [17]. Finally, we believe that the method will also be of interest for future clinical applications of x-ray CT, because DPC-based imaging can be carried out with standard x-ray tube sources $[14,18]$ and extended to fan-beam CT reconstruction schemes.

We gratefully acknowledge C. Grünzweig for the fabrication of the grating structures, the assistance of C. Kottler during the experiments, and T. Weitkamp for fruitful discussions.

[1] R. Fitzgerald, Phys. Today 53, No. 7, 23 (2000).

[2] A. Momose, Jpn. J. Appl. Phys. 44, 6355 (2005).

[3] U. Bonse and F. Busch, Prog. Biophys. Molec. Biol. 65, 133 (1996).

[4] C. Raven, A. Snigirev, I. Snigireva, P. Spanne, A. Souvorov, and V. Kohn, Appl. Phys. Lett. 69, 1826 (1996).

[5] F. Beckmann, K. Heise, B. Kolsch, U. Bonse, M. F. Rajewsky, and T. Biermann, Biophys. J. 76, 98 (1999).

[6] P. Cloetens, W. Ludwig, J. Baruchel, D. van Dyck, J. van Landuyt, J. P. Guigay, and M. Schlenker, Appl. Phys. Lett. 75, 2912 (1999)

[7] F. A. Dilmanian, Z. Zhong, B. Ren, X. Y. Wu, L. D. Chapman, I. Orion, and W.C. Thomlinson, Phys. Med. Biol. 45, 933 (2000).

[8] S. C. Mayo, T. J. Davis, T.E. Gureyev, P. R. Miller, D. Paganin, A. Pogany, A. W. Stevenson, and S. W. Wilkins, Opt. Express 11, 2289 (2003).

[9] P. J. McMahon, A. G. Peele, D. Paterson, J. J. A. Lin, T.H.K. Irving, I. McNulty, and K. A. Nugent, Opt. Commun. 217, 53 (2003).
[10] T. Weitkamp, A. Diaz, C. David, F. Pfeiffer, M. Stampanoni, P. Cloetens, and E. Ziegler, Opt. Express 13, 6296 (2005).

[11] A. Groso, M. Stampanoni, R. Abela, P. Schneider, S. Linga, and R. Müller, Appl. Phys. Lett. 88, 214104 (2006).

[12] A. Momose, W. Yashiro, Y. Takeda, Y. Suzuki, and T. Hattori, Jpn. J. Appl. Phys. 45, 5254 (2006).

[13] A. Bravin et al., Phys. Med. Biol. 52, 2197 (2007).

[14] F. Pfeiffer, T. Weitkamp, O. Bunk, and C. David, Nature Phys. 2, 258 (2006).

[15] F. Pfeiffer, M. Bech, O. Bunk, P. Kraft, E. F. Eikenberry, C. Brönnimann, C. Grünzweig, and C. David, Nature Mater. 7, 134 (2008).

[16] C. David, T. Weitkamp, F. Pfeiffer, A. Diaz, J. Bruder, T. Rohbeck, A. Groso, O. Bunk, M. Stampanoni, and P. Cloetens, Spectrochim. Acta, Part B 62, 626 (2007).

[17] F. Pfeiffer, O. Bunk, C. David, M. Bech, G. Le Duc, A. Bravin, and P. Cloetens, Phys. Med. Biol. 52, 6923 (2007).

[18] F. Pfeiffer, C. Kottler, O. Bunk, and C. David, Phys. Rev. Lett. 98, 108105 (2007).

[19] G. W. Faris and R. L. Byer, Appl. Opt. 27, 5202 (1988).

[20] Ya. I. Nesterets, T.E. Gureyev, and S. W. Wilkins, Appl. Phys. Lett. 89, 264103 (2006).

[21] T.E. Gureyev, Ya. I. Nesterets, and S.C. Mayo, Opt. Commun. 280, 39 (2007).

[22] A. C. Kak and M. Slaney, Principles of Computerized Tomography (IEEE, New York, 1987).

[23] In practice, where the projections are sampled with a finite resolution $\Delta y^{\prime}$, the filter is truncated at the Nyquist frequency $1 / 2 \Delta y^{\prime}$.

[24] These values for the refractive index correspond to polymethylmethacrylate $(\mathrm{C} 5 \mathrm{H} 8 \mathrm{O} 2)$ at a wavelength $\lambda=$ $0.5 \AA(E=25 \mathrm{keV})$.

[25] A. G. Ramm and A. I. Katsevich, The Radon Transform and Local Tomography (CRC Press, Boca Raton, 1996).

[26] F. Noo, R. Clackdoyle, and J.D. Pack, Phys. Med. Biol. 49, 3903 (2004).

[27] M. A. Anastasio and X. Pan, Opt. Lett. 32, 3167 (2007).

[28] We used the FReLoN 2000 (a fast-readout, low-noise CCD developed at the ESRF) with $1024 \times 1024$ pixels and a $28.0 \times 28.0 \mu \mathrm{m}^{2}$ pixel size (in the $2 \times 2$ binning mode). Because of the magnifying lens system, the effective pixel size in the recorded images was $16.1 \times$ $16.1 \mu \mathrm{m}^{2}$. For more details on the CCD, see, e.g., P. Coan et al., J. Synchrotron Radiat. 13, 260 (2006).

[29] For one projection, eight individual raw images (phase stepping) with exposure times of 0.4 seconds each were recorded. The DPC projections were obtained by extracting the phase of the first Fourier component after applying a one-dimensional discrete fast Fourier transform on the intensity values recorded during a phase-stepping scan in each detector pixel $[10,15,18]$.

[30] The interferometer was placed at a distance of $140 \mathrm{~m}$ from the wiggler source. The gratings were fabricated by a process involving photolithography, deep etching into silicon, and electroplating of gold. The first grating ( $\mathrm{Si}$ phase grating, G1) had a period of $p_{1}=3.99 \mu \mathrm{m}$ and a height of $h_{1}=31.7 \mu \mathrm{m}$. The corresponding values for the second grating (gold absorber grating, G2) were $p_{1}=$ $2.00 \mu \mathrm{m}$ and $h_{2}=30 \mu \mathrm{m}$. The distance between G1 and G2 was $361 \mathrm{~mm}$. See [17] for more details on the experimental setup. 